\title{
Prognostic significance of the sub-classification of stage pT3a renal tumors by perinephric and sinus fat invasion
}

\author{
DONGSHENG ZHU ${ }^{1 *}$, JINGYUAN CAO $^{2 *}, \mathrm{CHAO} \mathrm{ZHI}^{1 *}, \mathrm{TAO} \mathrm{GUO}^{1}$, \\ YUHONG LI ${ }^{3}$, ZHIQIANG LANG ${ }^{4}$ and GANG LI ${ }^{1}$ \\ ${ }^{1}$ Department of Urology, Tianjin Institute of Urology, The Second Hospital of Tianjin Medical University, Tianjin 300211; \\ ${ }^{2}$ Department of Urology, Binzhou Medical University Hospital, Binzhou, Shandong 256600; \\ ${ }^{3}$ Department of Pathology, The People's Hospital of Liaocheng, Liaocheng, Shandong 252000; \\ ${ }^{4}$ Department of Pathology, Yuhuangding Hospital of Qingdao University, Yantai, Shandong 264000, P.R. China
}

Received June 1, 2019; Accepted November 13, 2019

DOI: $10.3892 / \mathrm{ol} .2020 .11281$

\begin{abstract}
In the current Tumor-Node-Metastasis (TNM) classification system for renal cell carcinoma (RCC), both perinephric fat invasion (PFI) and renal sinus fat invasion (SFI) are classified at the T3a stage. However, their associated prognoses are clinically controversial. The present study proposes a new sub-classification criterion for pathological T3a (pT3a) RCC with SFI or PFI to resolve this dispute. Data were collected from consecutive records of 2,765 patients with T1a renal cancer, who had undergone partial nephrectomy (PN) between 2001 and 2015 at one of four hospitals. Among these patients, 127 cases were diagnosed with stage pT3a RCC with SFI or PFI, according to final pathological examination. The pathological characteristics, clinical data and follow-up observations were analyzed. Of the 127 patients, with an average follow-up duration of 56 months (range, 15-60 months), 17 cases of tumor recurrence were found. After analysis of the pathological findings, the following new sub-classification criteria was proposed for pT3a RCC with SFI or PFI: i) Type A, renal tumor invades the pseudo-capsule and contacts with the perinephric adipose tissues directly (3 recurrences out of 57 patients); ii) type $\mathrm{B}$, tumor protrudes into the perinephric adipose tissues like a tongue (4 recurrences out of 29 patients); and iii) type $C$, tumor nodules distribute in perinephric adipose tissues (10 recurrences out of 41 patients). There was statistically significant difference between the
\end{abstract}

Correspondence to: Dr Gang Li or Dr Dongsheng Zhu, Department of Urology, Tianjin Institute of Urology, The Second Hospital of Tianjin Medical University, 23 Pingjiang Road, Tianjin 300211, P.R. China

E-mail: drgangli2001@sina.com

E-mail: zhudongsheng@tmu.edu.cn

*Contributed equally

Key words: small renal cell carcinoma, renal cell carcinoma, T3a, specimen sampling three subtypes in terms of recurrence rate $(\mathrm{P}=0.023)$. In conclusion, controversies remain in the current TNM classification system for pT3a RCC. The present study added to the available data and found that pT3a RCC with tumor nodules in perinephric adipose or/and with an irregular tumor protruding into adipose tissues showed a higher recurrence rate. Thus, it is recommended that pT3a RCC should be carefully analyzed and should be considered differently to other stages of RCC.

\section{Introduction}

Renal cell carcinoma (RCC) accounts for 2-3\% of all malignancies, with an annual increase of $2 \%$ in the last 20 years globally (1). Most new cases of RCC are identified incidentally, and the improved detection is attributed to the advancement in imaging examinations, such as ultrasound (US) and computed tomography (CT). The vast majority of newly diagnosed cases are small renal cell carcinoma, which is classified clinically as stage T1a (cT1a) (2). The cT1a RCC is commonly associated with favorable treatment outcomes, therefore, nephron-sparing techniques, such as partial nephrectomy (PN), are recommended by the European Association of Eurology guidelines on RCC (3). However, among those patients who undergo PN for cT1a RCC, some may have their tumor upstaged to pT3a, according to the final pathological examination results (4), which causes a dilemma for surgeons. On the basis of the 2009 Tumor-Node-Metastasis (TNM) classification system, both perinephric fat invasion (PFI) and renal sinus fat invasion (SFI) are defined as stage T3a for RCC (5). However, there are still controversies with regard to the prognosis of patients with T3a RCC. An increasing number of studies have reported that patients with stage T3a RCC and SFI have a significantly worse prognosis compared with those with PFI (6-8). By contrast, other studies have suggested no significant differences in survival rates between patients with the two types of T3a RCC $(9,10)$. Thus, further investigations are required in order to clarify the cause underlying the controversy. The present study proposes a new sub-classification criterion for pT3a RCC with SFI or PFI, in order to provide new insights on this dispute. 


\section{Patients and methods}

Patients. A retrospective analysis of the nephrectomy database at The Second Hospital of Tianjin Medical University (Tianjin, China), Binzhou Medical University Hospital (Binzhou, China) and The People's Hospital of Liaocheng (Liaocheng, China) and Yuhuangding Hospital of Qingdao University (Yantai, China) was performed, after obtaining approval from the respective Institutional Review Boards. In total, 2,765 patients were included; each patient underwent PN for cTla RCC between December 2001 and December 2015, and were diagnosed by US or CT methods. The patients were excluded if they had familial or hereditary RCC, solitary kidney RCC or synchronous RCC. Finally, a total of 127 cases were recruited for this cohort. All patients provided written informed consent, and this study was conducted in accordance with the Declaration of Helsinki.

Clinicopathological analysis. Following PN, surgical specimens were fixed with $10 \%$ formalin for $24 \mathrm{~h}$ at room temperature. Subsequently, ink was used to stain the edges of the specimens for one minute at room temperature, and was fixed using $4 \%$ paraffin. The RCC specimens were routinely sliced at a thickness of $\sim 3 \mu \mathrm{m}$ and stained with hematoxylin-eosin $10 \mathrm{~min}$ at room temperature. The pathological examination was carried out by two professional genitourinary pathologists using and light microscope. The sections were classified according to the 2009 TNM classification system.

Follow-up observation. Postoperative follow-up was conducted based on the analysis of kidney and chest CT images 6 months after surgery, and then annually until 5 years after surgery, followed by one examination every 2 years. Magnetic resonance imaging or bone scans were applied when clinically indicated. Moreover, local recurrence of tumors, regional lymph nodes, and distant tissue and organ metastases were monitored.

Identification of sample size. Based on our pilot data, the sample size was estimated at a study power of $75 \%$ and a significance level of 5\% using the PASS11 software (NCSS, LLC). A sample size of at least 56 patients per group was suggested.

Statistical analysis. Pearson's $\chi^{2}$ and Fisher exact text were performed to assess the categorical variables. The numerical variables were analyzed using the Mann-Whitney U test. Kaplan-Meier curves were plotted to estimate recurrence-free survival probabilities, using the log-rank test. Multivariate cox regression analysis was also applied to identify predictors of recurrence. All statistical analyses were performed using the SPSS20 software (IBM Corp.). $\mathrm{P}<0.05$ was used to indicate a statistically significant difference.

\section{Results}

In total, 1,659 males and 1,106 females with a median age of 63 years (range, 38-82 years) underwent PN for a solitary cT1 RCC. According to the pathological examination results, 127 cases were upstaged to pT3a, showing a rate of $4.59 \%$, including 55 SFI cases and 72 PFI cases. The characteristics of the 127 selected patients are listed in Table I. Based on the analysis of the sample sections that were pathologically determined as cT1a, a new sub-classification criteria for pT3a RCC with SFI or PFI was generated: i) Type A, renal tumor invades the pseudo-capsule and is in contact with the perinephric adipose tissues directly (Fig. 1); type B, the tumor protrudes into the perinephric adipose tissues like a tongue (Fig. 2); type $\mathrm{C}$, tumor nodules distribute in perinephric adipose tissues (Fig. 3).

There were 57, 29 and 41 patients in the three subtypes, respectively, with 3 (1 with SFI and 2 with PFI), 4 (2 with SFI and 2 with PFI) and 10 (4 with SFI and 6 with PFI) cases of recurrences during follow-up. Notably, 2 patients demonstrated distant metastases, both of which were of type C, showing a statistically significant difference in the recurrence rate $(\mathrm{P}=0.023$; Fig. 4$)$.

Kaplan-Meier curves also demonstrated consistent results of the recurrence rates $(\mathrm{P}=0.015$; Fig. 5$)$. For $\mathrm{PN}$, the difference in the recurrence rates of SFI and PFI in the 3 types was not statistically significant (Fig. S1). In the multivariate cox regression analyses for predictors of disease recurrence adjusted for age, sex, surgery approach, tumor size, histology, Fuhrman grade (11), margin status and the sub-classification presented in this study, the occurrence was independently associated with sub-classification $(\mathrm{P}=0.021$; Tables II; and $\mathrm{P}=0.009$; $\mathrm{SI})$.

\section{Discussion}

It is recognized that both PFI and SFI belong to the T3a stage in RCC, since the preoperative imaging examination cannot distinguish the micro-tumor invasion of perinephric fat. Srivastava et al (12) assessed 28,854 RCC patients undergoing PN, and found that $4.2 \%$ of patients with cT1a tumors were upstaged to pT3a between 1998 and 2003. A number of other studies reported a similar value, such as those by Gorin et al (13), Weight et al (14) and Jeong et al (15), which reported the rates of $3.9,4.8$ and $5.7 \%$, respectively. The rate in the present study was $4.5 \%$, which was similar to that of the aforementioned studies. It is apparent that although the pathological upstaging of RCC after surgery is rare, it still occurs, and thus, further studies on this phenomenon are required.

Only a few relevant studies could be retrieved on the two types of pathological findings of fat invasion (SFI and PFI) in T3a RCC. Bonsib et al (16) suggested that SFI could lead to a worse prognosis, from an anatomical perspective. The reasons were as follows: Firstly, there is no renal capsule surrounding the renal sinus. As the capsule serves as a barrier to extra-renal spread, tumors with SFI are more likely to spread. Secondly, the renal sinus is rich in veins and lymphatic vessels. Conversely, the density of micrangium and lymphatics in perinephric fat is much lower than that in the sinus fat, and thus, the occurrence of SFI increases the opportunity for tumor invasion. Another study involving 205 patients with RCC, including 162 patients with PFI and 43 patients with SFI, reported death rates of 59\% $(\mathrm{n}=95)$ and $72 \%(n=31)$ during follow-up, respectively. This indicated that the occurrence of SFI was more likely to cause death from RCC compared with that of PFI [risk ratio (RR), 1.63; 95\% CI, 1.09-2.46; $\mathrm{P}=0.018)$ ] (6). However, Margulis et al (10) found that there was no difference between SFI and PFI 
Table I. Patient characteristics $(n=127)$.

\begin{tabular}{|c|c|c|c|}
\hline Variable & SFI & PFI & P-value \\
\hline Patients, $\mathrm{n}$ & 55 & 72 & \\
\hline Sex, n & & & 0.37 \\
\hline Male & 27 & 42 & \\
\hline Female & 28 & 30 & \\
\hline Age, years & & & 0.62 \\
\hline Median & 64 & 61 & \\
\hline IQR & $38-79$ & $49-82$ & \\
\hline Surgical approach, n & & & 0.26 \\
\hline Open & 22 & 21 & \\
\hline Laparoscopic & 33 & 51 & \\
\hline Pathological tumor size, $\mathrm{cm}$ & & & 0.22 \\
\hline Median & 3.2 & 3.5 & \\
\hline IQR & $2.1-3.7$ & $2.5-3.8$ & \\
\hline Laterality, n & & & 0.86 \\
\hline Left & 30 & 37 & \\
\hline Right & 25 & 35 & \\
\hline Tumor histological type, $\mathrm{n}$ & & & 0.72 \\
\hline Clear cell & 30 & 36 & \\
\hline Non-clear cell & 25 & 36 & \\
\hline R.E.N.A.L. score ${ }^{a}$ & & & 0.13 \\
\hline Median & 6.2 & 5.5 & \\
\hline IQR & $4-7$ & $4-6$ & \\
\hline Fuhrman grade, $n$ & & & 0.85 \\
\hline Low (I-II) & 20 & 25 & \\
\hline High (III-IV) & 35 & 47 & \\
\hline Margin status, $\mathrm{n}$ & & & 0.73 \\
\hline Positive & 3 & 6 & \\
\hline Negative & 52 & 66 & \\
\hline
\end{tabular}

SFI, renal sinus fat invasion; PFI, perinephric fat invasion; IQR, interquartile range; ${ }^{a}$ According to Kutikov A, Uzzo RG: The R.E.N.A.L. nephrometry score: a comprehensive standardized system for quantitating renal tumor size, location and depth. The Journal of urology 2009, 182(3): 844-853. R.E.N.A.L.: R, radius (tumor size as maximal diameter); E, exophytic/endophytic properties of the tumor; N, nearness of tumor deepest portion to the collecting system or sinus; A, anterior/posterior descriptor; and L, location relative to the polar line.

with regard to 5-year cancer-specific survival rate (50.8 and $54.1 \%$, respectively; $\mathrm{P}=0.782$ ). The study involved a total of 365 patients with pT3a RCC, among whom 166 cases were diagnosed with SFI and 199 with PFI, after a mean follow-up time of 33.5 months (range, 6.1-158.6 months). Patients with SFI and PFI therefore had similar clinical and pathological characteristics, and tumors with SFI were more likely to involve the urothelial collecting system, due to the proximity to the renal pelvis, rather than distant metastasis.

The cause of conflicting results should be further explored, as there may be other factors contributing to the different outcomes. In the present study, after thorough examination of the pathological findings by microscopy, T3a RCC fat invasion with SFI and PFI was divided into three subtypes, as

Table II. Multivariate Cox regression analysis for predictors of disease recurrence.

\begin{tabular}{lccc}
\hline Variable & HR & $\begin{array}{c}95 \% \text { Confidence } \\
\text { interval }\end{array}$ & P-valu \\
\hline Sex & & &
\end{tabular}

Sex

\section{Female}

Male

Age

Laterality

Left

Right

Surgical approach

Open

Laparoscopic

Tumor size

Histological type

Non-clear cell

Clear cell

Fuhrman grade

Low (I-II)

High (III-IV)

Margin status

Negative

Positive

Sub-classification

Type A
Type B
Type C

Type A

Type $\mathrm{C}$

HR, hazard ratio; Ref., reference.

Ref.

0.970

Ref.
0.947

$0.660-1.358$

0.767

1.001

0.984-1.018

0.926

Ref.

$0.956 \quad 0.663-1.379$

0.811

Ref.

1.084

0.737-1.594

0.682

1.073

$0.500-2.303$

0.856

Ref.

$1.093 \quad 0.757-1.577$

0.635

Ref.

$0.935 \quad 0.641-1.364$

0.728

Ref.

$0.977 \quad 0.491-1.944$

0.947

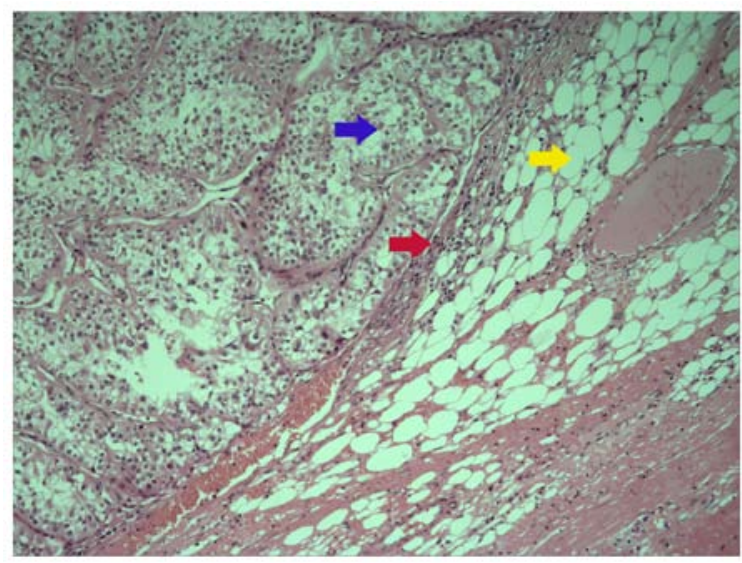

Figure 1. Sub-classification criteria type A: Renal tumor invades the pseudocapsule and is in contact with the perinephric adipose tissues directly. Blue arrow, tumor; red arrow, pseudo-capsule; yellow arrow, adipose tissue. Hematoxylin-eosin staining. Mgnification, x200.

aforementioned. We speculate that the aforementioned conflicts may be attributed to failure to distinguish between these three situations. 


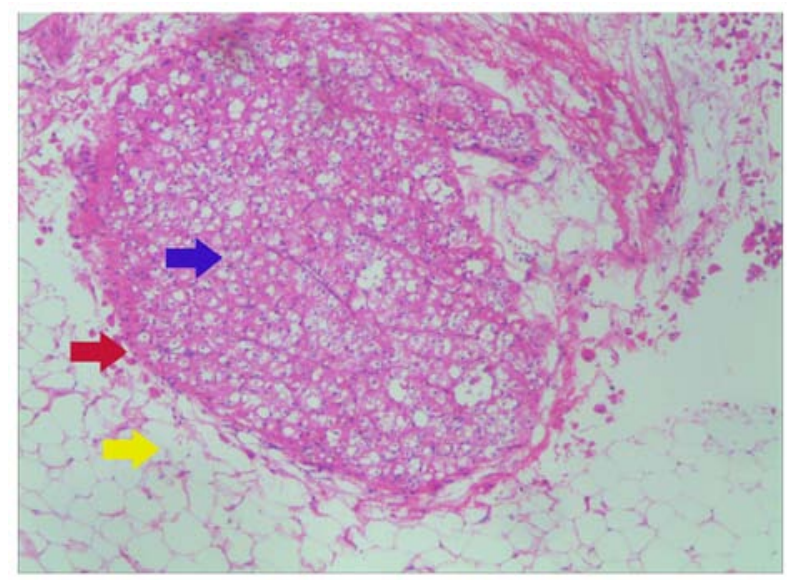

Figure 2. Sub-classification criteria type B: The tumor protrudes into the perinephric adipose tissues like a tongue. Blue arrow, tumor; red arrow, pseudocapsule; yellow arrow, adipose tissue. Hematoxylin-eosin staining. Magnification, $\mathrm{x} 200$.

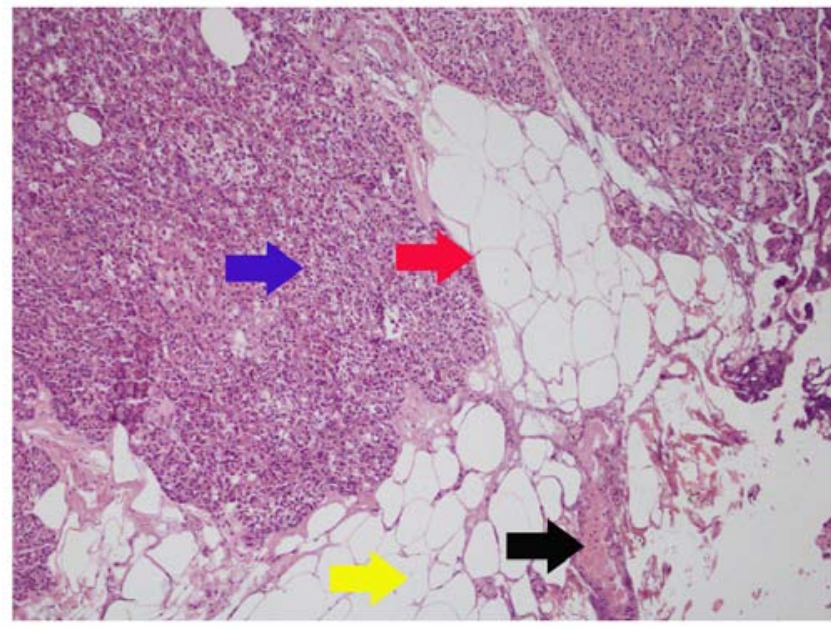

Figure 3. Sub-classification criteria type C: Tumor nodules distributed in perinephric adipose tissues. Blue arrow, tumor; red arrow, pseudo-capsule; yellow arrow, adipose tissue; black arrow, tumor nodules like an island. Hematoxylineosin staining. Magnification, x200.

The first subtype is characterized by a tumor that invades the pseudo-capsule and contacts with the perinephric adipose tissues directly. In the present study, among the 127 patients, 57 were classified as this subtype. During the follow-up, only 3 patients relapsed. Consequently, if the adipose tissue covering the tumor surface can be removed during $\mathrm{PN}$, the tumor can be removed completely and the recurrence rate could be reduced. A previous study, including 1,367 patients with small RCC, reported the 5-year recurrence-free survival rates of patients with pT1a RCC and pT3a RCC as 98.0 and $95.2 \%$, respectively $(\mathrm{P}=0.521)(17)$. Thus, patients with pT3a $\mathrm{RCC}$ at the pT1a stage have similar oncological outcomes following PN, suggesting that PN is suitable for T3a RCC (17). Oh et al (18) analyzed the records of 3,567 patients who underwent PN and the observations made during a median 43-month follow-up period, and found that PN offers similar recurrence-free survival outcomes compared with radical nephrectomy in patients with cT1a but not pT3a RCC. In the

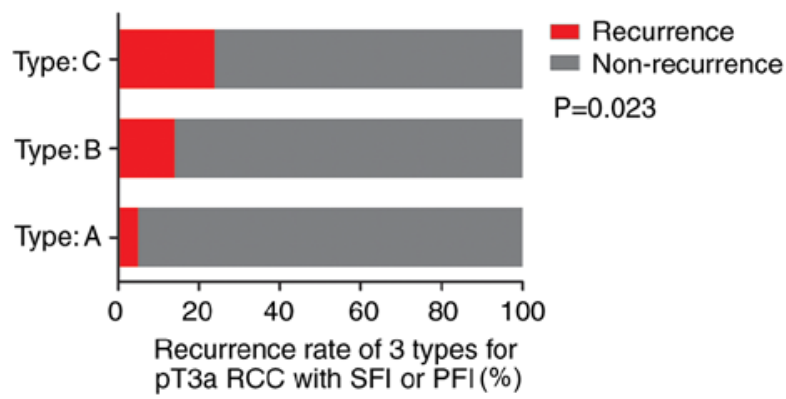

Figure 4. Recurrence rates of the three types (A, B and C) of pT3a RCC with SFI or PFI. $\mathrm{P}=0.023$, as determined by $\chi^{2}$ test. RCC, renal cell carcinoma; $\mathrm{SFI}$, renal sinus fat invasion; PFI, perinephric fat invasion.

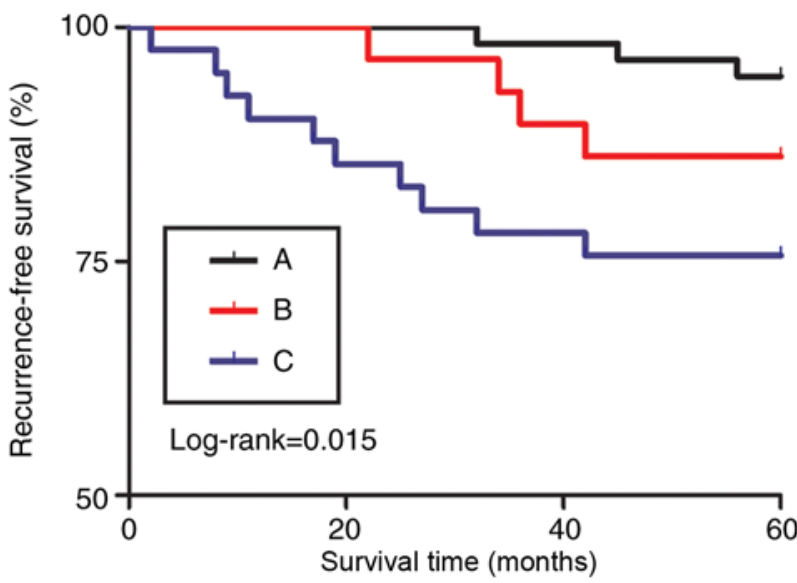

Figure 5. Recurrence-free survival rates of the three types (A, B and C) of pT3a renal cell carcinoma after partial nephrectomy.

present study, the T3a RCC classified into type A may also be attributed to the possibility of $\mathrm{PN}$ in T3a RCC reported in aforementioned literatures. Whether it is SFI or PFI, the tumor can be removed completely during PN.

Renal tumors that have protruded into the perinephric adipose tissues like a tongue are defined as the second subtype. During surgery, if the surface adipose tissues are improperly removed, the residual tumor that has protruded into the surrounding fat may lead to the occurrence of a positive surgical margin, which may result in tumor recurrence and even tumor metastasis (19). Therefore, during PN, surgeons should not excessively remove the fat on the surface of the tumor to prevent the positive surgical margin, particularly in the case of irregularly shaped tumors.

The third pathological subtype is a rare type characterized by tumor nodules that are distributed in the perinephric adipose tissues. The tumor nodules are like islands distributed among the perinephric or the renal sinus adipose tissues. After $\mathrm{PN}$, despite the complete removal of adipose tissues covering the kidney surface, it is still difficult to completely remove the tumor nodules that are scattered in the perinephric fat, as they are invisible to the naked eye. Thus, a radical resection should be performed for this subtype. However, since the tumor nodules cannot be detected by imaging before surgery, cTla RCC is commonly treated by PN. Therefore, close follow-up is recommended with the upstaging of pT3a RCC in this type. Radical 
surgery should be performed immediately upon the recurrence after surgery.

The present study has several potential limitations. The study is retrospective with a relatively small sample size from four institutions. Prospective, large sample size studies are required for further research. Secondly, no differences were observed between low-grade (grades 1-2) and high-grade (grades 3-4) tumors in the present study $(\mathrm{P}=0.728)$, although another study has demonstrated similar results (17). The most widely used RCC assessment is the Fuhrman grading system (11) released in 1982. Although widely used, the grading system was based solely on the analysis of 103 renal cancer cases, of which only 85 were followed up, and did not consider the histological classification of the RCC. In practical applications, the grading system has problems, such as difficulty in interpretation and poor repeatability. Therefore, the system was replaced by the International Society of Urologic Pathologists (ISUP) grade in 2016 (20). The ISUP grade will be used in future studies. Thirdly, due to the small sample size, tumors were broadly divided into clear cell and 'non-clear cell' categories, which may introduce bias.

Regardless of the aforementioned limitations, the present study demonstrated the association between the classification of fat invasion in T3a RCC and tumor recurrence after $\mathrm{PN}$, for cT1a RCC upstaging to pT3a. We recommend that the pathological examination results of patients with RCC should be carefully evaluated and patients with pT3a RCC should be assessed again individually with regards to fat invasion.

\section{Acknowledgements}

Not applicable.

\section{Funding}

This study was supported by the Tianjin Municipal Natural Science Foundation (grant no. 17JCYBJC26000) and the Shandong Provincial Medical Health Technology Development Project (grant no. 2014WS0189).

\section{Availability of data and materials}

The datasets used and/or analyzed during the current study are available from the corresponding author on reasonable request.

\section{Authors' contributions}

GL and DSZ conceived and directed the project. DSZ contributed to the writing of the manuscript. DSZ, GL and TG analyzed the data. DSZ, JYC, YHL, CZ and ZQL collected the clinical data. All authors read and approved the final manuscript.

\section{Ethics approval and consent to participate}

The study protocols were approved by the Ethical Committee Review Board of the Second Hospital of Tianjin Medical University (Tianjin, China). All participants provided written informed consent.

\section{Patient consent for publication}

Not applicable.

\section{Competing interests}

The authors declare that they have no competing interests.

\section{References}

1. Ferlay J, Steliarova-Foucher E, Lortet-Tieulent J, Rosso S, Coebergh JW, Comber H, Forman D and Bray F: Cancer incidence and mortality patterns in Europe estimates for 40 countries in 2012. Eur J Cancer 49: 1374-1403, 2013.

2. Laguna MP, Algaba F, Cadeddu J, Clayman R, Gill I, Gueglio G, Hohenfellner M, Joyce A, Landman J, Lee B, et al: Current patterns of presentation and treatment of renal masses A clinical research office of the endourological society prospective study. J Endourol 28: 861-170, 2014

3. Ljungberg B, Bensalah K, Canfield S, Dabestani S, Hofmann F, Hora M, Kuczyk MA, Lam T, Marconi L, Merseburger AS, et al: EAU guidelines on renal cell carcinoma 2014 update. Eur Urol 67: 913-924, 2015.

4. Ramaswamy K, Kheterpal E, Pham H, Mohan S, Stifelman M, Taneja S and Huang WC: Significance of pathologic T3a upstaging in clinical T1 renal masses undergoing nephrectomy. Clin Genitourin Cancer 13: 344-349, 2015.

5. Martinez-Salamanca JI, Huang WC, Millan I, Bertini R, Bianco FJ, Carballido JA, Ciancio G, Hernández C, Herranz F, Haferkamp A, et al: Prognostic impact of the 2009 UICC/AJCC TNM staging system for renal cell carcinoma with venous extension. Eur Urol 59: 120-127, 2011.

6. Thompson RH, Leibovich BC, Cheville JC, Webster WS, Lohse CM, Kwon ED, Frank I, Zincke H and Blute ML: Is renal sinus fat invasion the same as perinephric fat invasion for pT3a renal cell carcinoma. J Urol 174: 1218-1221, 2005.

7. Chen K, Lee BL, Huang HH, Tan BY, Lee LS, Ng LG, Lau W and Yuen JS: Tumor size and Fuhrman grade further enhance the prognostic impact of perinephric fat invasion and renal vein extension in T3a staging of renal cell carcinoma. Int J Urol 24: 51-58, 2017.

8. Zhang Z, Yu C, Velet L, Li Y, Jiang L and Zhou F: The difference in prognosis between renal sinus fat and perinephric fat invasion for pT3a renal cell carcinoma A meta-analysis. PLoS One 11: e0149420, 2016.

9. Mouracade P, Dagenais J, Chavali JS, Kara O, Nelson RJ, Maurice MJ, Reese J, Rini BI and Kaouk JH: Perinephric and sinus fat invasion in stage pT3a tumors managed by partial nephrectomy. Clin Genitourin Cancer 16: e1077-e1082, 2018.

10. Margulis V, Tamboli P, Matin SF, Meisner M, Swanson DA and Wood CG: Location of extrarenal tumor extension does not impact survival of patients with pT3a renal cell carcinoma. J Urol 178: 1878-1882, 2007.

11. Fuhrman SA, Lasky LC and Limas C: Prognostic significance of morphologic parameters in renal cell carcinoma. Am J Surg Pathol 6: 655-663, 1982

12. Srivastava A, Patel HD, Joice GA, Semerjian A, Gorin MA, Johnson MH, Allaf ME and Pierorazio PM: Incidence of T3a up-staging and survival after partial nephrectomy Size-stratified rates and implications for prognosis. Urol Oncol 36: 12.e7-12.e13, 2018 .,

13. Gorin MA, Ball MW, Pierorazio PM, Tanagho YS, Bhayani SB, Kaouk JH, Rogers CG, Stifelman MD, Khalifeh A, Kumar R, et al: Outcomes and predictors of clinical T1 to pathological T3a tumor up-staging after robotic partial nephrectomy A multi-institutional analysis. J Urol 190: 1907-1911, 2013.

14. Weight CJ, Lythgoe C, Unnikrishnan R, Lane BR, Campbell SC and Fergany AF: Partial nephrectomy does not compromise survival in patients with pathologic upstaging to pT $2 / \mathrm{pT} 3$ or high-grade renal tumors compared with radical nephrectomy. Urology 77: 1142-1146, 2011.

15. Jeong SH, Kim JK, Park J, Jeon HJ, Yoon MY, Jeong CW, Ku JH, Kim HH and Kwak C: Pathological T3a upstaging of clinical T1 renal cell carcinoma outcomes according to surgical technique and predictors of upstaging. PLoS One 11: e0166183, 2016.

16. Bonsib SM, Gibson D, Mhoon M and Greene GF: Renal sinus involvement in renal cell carcinomas. Am J Surg Pathol 24: 451-458, 2000. 
17. Lee C, You D, Yoo S, Song C, Hong B, Hong JH, Ahn H and Kim CS: Oncological outcomes of patients with incidental pathological T3a stage small renal cell carcinoma after partial nephrectomy. J Cancer Res Clin Oncol 142: 1651-1657, 2016.

18. Oh JJ, Byun SS, Lee SE, Hong SK, Lee ES, Kim HH, Kwak C, $\mathrm{Ku} \mathrm{JH}$, Jeong CW, Kim YJ, et al: Partial nephrectomy versus radical nephrectomy for non-metastatic pathological $\mathrm{T} 3 \mathrm{a}$ renal cell carcinoma A multi-institutional comparative analysis. Int J Urol 21: 352-357, 2014.
19. Park M, Shim M, Kim M, Song C, Kim CS and Ahn H: Prognostic heterogeneity in T3aNOM0 renal cell carcinoma according to the site of invasion. Urol Oncol 35: 458.e17-458.e22, 2017.

20. Moch H: The WHO/ISUP grading system for renal carcinoma. Der Pathologe 37: 355-360, 2016 (In German).

(i)@ $\ominus$ This work is licensed under a Creative Commons Attribution-NonCommercial-NoDerivatives 4.0 International (CC BY-NC-ND 4.0) License. 